\title{
Legalización de la marihuana, la libertad que amenaza la salud pública
}

\author{
V. Venegas
}

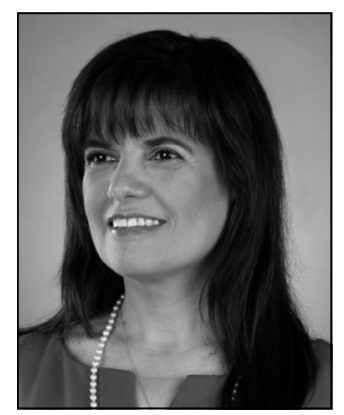

La marihuana, es un término genérico utilizado para denominar a "Cannabis Sativa", especie herbácea con propiedades psicoactivas. Existen antecedentes de uso de C. Sativa que datan de 4.000 años A.C. en China, para tratar diversas afecciones. En USA estuvo disponible su venta en farmacias sin receta hasta 1941 y en 1970 la ley de sustancias controladas la declara ilegal.

C. Sativa tiene aproximadamente 489 componentes con más de 80 compuestos terpenofenólicos llamados cannabinoides. El resto son componentes como terpenos, hidrocarburos, cetonas, aldehídos y otros, hidrófobos pequeños capaces de cruzar la barrera hematoencefálica. En 1963 y 1964 se descubren estructuras químicas del Delta-9-Tetrahidrocannabinol ( $\triangle 9$-THC) componente psicoactivo de Cannabis y Cannabidiol (CBD), los dos compuestos principales de Cannabis. La variabilidad de cepas se da por el índice $\Delta 9-\mathrm{THC} / \mathrm{CBD}$. La proporción de $\triangle 9-\mathrm{THC}$ se ha incrementado en el tiempo desde $1-5 \%$ a fines 1960 s a rangos actuales de $10-15 \%$, por tanto mayor potencia psicoactiva. Los receptores de cannabinoides (CB1 y CB2) ligan endocannabinoides. Estos se producen durante la excitación neuronal excesiva como parte de un circuito de retroalimentación negativa natural en neuronas gabaérgicas y glutamaérgicas. Este efecto no es completamente predecible, explicaría el meca- nismo de control y a la vez de exacerbación de crisis epilépticas. Es importante conocer que se han desarrollado estimulantes cannabinoides sintéticos.

Receptores CB1 cerebrales están ampliamente distribuidos en corteza prefrontal, responsable de funciones ejecutivas, motivación, planificación, control de actitudes y selección de alternativas en la toma de decisiones. En el hipocampo y estriado se relacionan con la búsqueda de incentivos, recompensa, procesos de memoria y aprendizaje ${ }^{1}$.

La vía más común de uso de Cannabis es la inhalada. Las hojas gris-verdoso trituradas y flores de $C$. Sativa se fuman en cigarrillos o pipas. El hachís es un producto de la resina de las flores, que puede ser inhalado o ingerido. También se utilizan infusiones y extractos de aceite que pueden ser mezclados en diversos productos alimenticios. Tiene alta liposolubilidad, alta unión a proteínas plasmáticas y gran volumen de distribución, con rápida distribución a cerebro, tejido adiposo y otros órganos.

El consumo provoca sensación de euforia, disminuye la ansiedad y el estado de alerta. Los consumidores primerizos e individuos psicológicamente vulnerables pueden experimentar ansiedad, disforia, y pánico. Se puede alterar la percepción sensorial (visión de colores más brillantes, música más intensa, percepción espacial distorsionada, percepción tempo-

Recibido el 1 de diciembre de 2014. Aceptado el 1 de diciembre de 2014.

\footnotetext{
Viviana Venegas Silva $(\triangle)$

Neuróloga Pediátrica.

Presidenta de la Sociedad de Neurología de la Infancia y Adolescencia (SOPNIA).

E-mail: vivi.venegas.s@gmail.com
} 
ral más rápida que lo real), aumenta el tiempo de reacción, deteriora atención, concentración, memoria a corto plazo y la capacidad de evaluación de riesgos. El efecto agudo deteriora coordinación psicomotora e interfiere la capacidad de realizar tareas complejas que requieren atención dividida como la conducción. El deterioro psicomotor, de cognición, coordinación y juicio dura más horas que los cambios del estado de ánimo percibidos por el consumidor, debido a la acumulación en el tejido adiposo y la liberación lenta de THC. En dosis altas provoca alucinaciones, pensamiento místico, despersonalización, grandiosidad transitoria, paranoia y otros síntomas de psicosis. Estos efectos son aditivos cuando se usa Cannabis en combinación con otros depresores del sistema nervioso central como el alcohol.

La marihuana para fines medicinales y/o recreativos se ha legalizado en varios países, por lo que los médicos son cada vez mas consultados sobre sus potenciales efectos adversos y beneficiosos para la salud. Se consideran productos para fines medicinales aquellos fitofármacos con altos índices CBD:THC. La FDA ha permitido tratar alrededor de 125 niños con epilepsia refractaria con productos derivados de Cannabis y actualmente se están enrolando pacientes para estudios multicéntricos internacionales tanto en epilepsia refractaria como en otras dolencias. Cannabis se ha empleado sin evidencia científica suficiente para tratar glaucoma, asma, cáncer, anorexia en enfermedades crónicas, migraña, insomnio, náuseas, vómitos asociados a quimioterapia, espasticidad en esclerosis múltiple, neuropatías periféricas y otros trastornos neuromusculares.

En Chile se ha generado una gran controversia, por la iniciativa de legalizar el consumo "medicinal, recreativo y sacramental de la marihuana y otras especies vegetales y fungis con efecto psicoactivo". No es casualidad que la comunidad científica médica nacional se haya pronunciado en contra. En Chile el consumo personal no está penalizado (Ley $20.000,2005$ ) como si ocurre en gran parte del mundo, entonces cuesta entender los esfuerzos para liberalizar aún más su uso. El espíritu del legislador contemplaba al consumidor como paciente, sin criminalizar el porte para consu- mo personal y privado. Legalizar significa dejar a la marihuana en el mismo estado que el alcohol y el tabaco, sujetos a "regulación", y sabemos lo que eso significa, con las altísimas cifras de consumo de alcohol y tabaco. Así, regular es un gran verbo, pero implementarlo efectivamente es muy difícil. El alcohol y tabaco son los dos primeros factores de riesgo para la salud física y psíquica en el mundo y también en Chile.

Es importante comprender que el problema de las drogas es multidimensional. El consumo de drogas es un problema de salud pública, y el microtráfico y tráfico son un problema de seguridad pública, esta diferenciación es fundamental y en política es necesario tratar de compatibilizar la salud pública con la seguridad pública. El fenómeno de las drogas es muy complejo, afecta la salud de consumidores, relaciones familiares, facilita delincuencia, impacta negativamente la percepción de seguridad y deteriora calidad de vida en barrios y ciudades. Las políticas de control en general se orientan en intentar disminuir el consumo, bajo concepciones de bienestar-salud y seguridad. No hay una solución única para el "problema de las drogas" en parte porque es difícil describir el problema, sus repercusiones sociales, en la salud y los diferentes contextos/ país. Criterios no científicos han encendido el debate durante muchos años.

Históricamente, las medidas de salud pública han tenido un efecto sobre la salud de las poblaciones en todo el mundo. La esperanza de vida se ha incrementado en forma espectacular en gran medida por la aplicación de medidas de salud pública encaminadas a mejorar el saneamiento, reducir la contaminación ambiental y prevenir enfermedades infecciosas y transmisibles (WHO, 1998). Sin embargo, aún cuando han disminuido las epidemias de enfermedades infecciosas y transmisibles, ha crecido en importancia los riesgos para la salud asociados con los modos de vida y las enfermedades crónicas como causas de mortalidad, lesiones, enfermedad y discapacidad. Esas intervenciones subrayan la necesidad de modificar el entorno y el comportamiento individual. Las inquietudes relacionadas con la justicia, la libertad, la moralidad y otras cues- 
tiones ajenas al campo de la salud ocupan un lugar importante en la elaboración de políticas de drogas y no debieran ser ignoradas por los expertos en salud pública ${ }^{2}$.

La legalización de la marihuana en Chile genera un dilema entre quienes consumen marihuana, representado principalmente por jóvenes provenientes de familias de ingresos medios y altos, para quienes la marihuana no genera una amenaza social, no es camino a delincuencia o fuente de desestabilización de sus estudios, ni familiar futura ni pone en riesgo su salud y bienestar, resienten que la justicia se inmiscuya en su consumo y restrinja el uso recreacional, considerándolo un derecho. Nunca se evoca la evidencia científica que demuestra el daño a la salud, por lo que se interpreta como excesiva la participación de la justicia en sus costumbres. Otra parte de la sociedad que no consume (la mayoría) y en particular para aquellos con escasas oportunidades, la droga significa disminuir sus opciones, la legalización podría validar el consumo, facilitar el acceso, disminuir la edad de inicio en adolescentes, disminución en precepción de riesgo y una probable normalización del uso. Existe actualmente una noción popular que la marihuana es inofensiva, por lo que el acceso no debiera ser considerado ilegal y así lo están planteando también algunos legisladores desde la mirada de los derechos individuales y la libertad de decidir.

Los principales riesgos a la salud asociados al uso de marihuana estudiados son ${ }^{3}$ :

Riesgo de adicción, la evidencia indica claramente que el consumo de marihuana a largo plazo puede conducir a la adicción. Aproximadamente un $9 \%$ de quienes experimentan con marihuana presentarán adicción, esta cifra sube a 1 de cada 6 casos que inician el consumo en la adolescencia y de 25 a $50 \%$ de adicción en quienes la consumen diariamente.

El consumo de marihuana en adolescentes es particularmente problemático, por la mayor vulnerabilidad reportada en todos los estudios de efectos adversos a largo plazo. Esto se relaciona con que el sistema endocannabinoide, presenta un desarrollo activo durante la adolescencia. El uso precoz y regular de marihuana predice mayor riesgo de adicción y un aumen- to en el riesgo de uso de otras drogas ilícitas. Quienes comienzan el consumo en adolescencia tienen aproximadamente 2 a 4 veces más probabilidades de tener dependencia a Cannabis dentro de 2 años después del primer uso ${ }^{4}$. El Servicio Nacional para la Prevención y Rehabilitación del Consumo de Drogas y Alcohol (SENDA) dio a conocer los resultados del $10^{\circ}$ Estudio Nacional de Drogas en Población Escolar realizado entre octubre y diciembre de $2013^{5}$, abarcó 58 mil estudiantes, evidenciando un importante aumento en el consumo de marihuana respecto a la misma medición del año 2011 , donde el $19,5 \%$ de los encuestados reconoció haber consumido marihuana al menos una vez en ese año y el 2013 la cifra aumentó a 30,6\%, con un mayor aumento en establecimientos particulares pagados $(14,6 \%$ versus $26,2 \%$ ) Esta alza es consistente con una disminución en la percepción de riesgo (48\% el 2011 y 21,1\% el 2013).

En Chile el 20\% de los consumidores de marihuana son consumidores problemáticos. En el $60 \%$ de los adolescentes en tratamiento por drogas, la droga principal de consulta es marihuana. En adolescentes infractores de ley en tratamiento la marihuana representa el 39\% y pasta base el $33 \%$.

Riesgos sobre el desarrollo cerebral, trans curre en forma activa y continua desde el período prenatal hacia la adolescencia, modelado significativamente por la experiencia y las agresiones ambientales tales como la exposición a $\Delta^{9}$-THC. En estudios en animales se ha demostrado que la exposición temprana a $\Delta^{9}$-THC puede sensibilizar el sistema del circuito de la recompensa el cual es esencial para el aprendizaje y contribuye a la vinculación entre una conducta y sus consecuencias. La exposición prenatal interfiere con la dinámica del citoesqueleto, que es crítico para el establecimiento de conexiones axonales entre neuronas. En comparación con controles no expuestos, el consumo regular de marihuana durante la adolescencia, presentan conectividad neuronal alterada (menos fibras) en regiones como precuneus, zona clave por sus conexiones implicadas en funciones con un alto grado de integración (estado de alerta y conciencia) y la fimbria, área del hipocampo importante en 
aprendizaje y memoria. La conectividad funcional reducida también ha sido reportada en redes prefrontales y redes subcorticales.

Hay regiones del cerebro más vulnerables que otras a los efectos a largo plazo de la marihuana y este efecto negativo en la conectividad cerebral es más prominente si se inicia en la adolescencia o adulto joven, lo que puede ayudar a explicar el hallazgo de una asociación entre el uso frecuente de marihuana y disminuciones significativas del coeficiente intelectual. La vulnerabilidad del adolescente es neurobiológica, psicológica y social. Una cohorte de adolescentes menores de 17 años con uso diario de Marihuana versus sin consumo, seguidos y comparados hasta los 30 años $(\mathrm{n}=2.537$ $v s \mathrm{n}=3.765$ ), encontró menor probabilidad de término de escuela secundaria y de grado universitario, alta probabilidad de dependencia a marihuana y de uso de sustancias ilícitas posteriormente, además de mayor probabilidad de intento de suicidio en el grupo consumidor ${ }^{6}$.

Datos preclínicos sugieren que el consumo de marihuana en la adolescencia es la puerta de entrada a otras drogas e influye en múltiples conductas adictivas en la edad adulta. En modelos animales de roedores expuestos a cannabinoides durante la adolescencia, hay una disminución de la reactividad de la dopamina en neuronas que modulan la recompensa en regiones del cerebro. Este efecto podría explicar el aumento de la susceptibilidad al abuso adicción a varias drogas más tarde en la vida, como se ha informado en la mayoría de los estudios epidemiológicos.

En relación a riesgo de enfermedades mentales, el uso regular de marihuana se asocia con mayor riesgo de ansiedad y depresión. Se vincula con psicosis (incluyendo esquizofrenia), especialmente en casos con vulnerabilidad genética donde la marihuana puede afectar negativamente el curso de la enfermedad. Es difícil de establecer causalidad en estos estudios porque los factores que no sean el consumo de marihuana (psicosociales) pueden estar asociados directamente con el riesgo de enfermedad mental, predisponiendo tanto al consumo de marihuana y enfermedad mental.

En cuanto a los efectos sobre rendimiento escolar y escolaridad, en un estudio publicado el 2013 el 6,5\% de los estudiantes de último curso de secundaria informó consumo diario o casi a diario de marihuana; el sesgo de este estudio es que excluye a los jóvenes desertores del colegio grupo que podrían tener tasas más altas de consumo. El uso de marihuana perjudica funciones cognitivas, así muchos estudiantes podrían estar funcionando en un nivel cognitivo por debajo de su capacidad natural por períodos de tiempo considerables después de consumir el fin de semana. Un estudio chileno realizado por A. Dörr comparó el rendimiento en 4 pruebas neuropsicológicas, encontró peor rendimiento en todas las pruebas en el grupo de adolescentes consumidores versus los no consumidores de marihuana. Los jóvenes no consumidores tenían una significativa mayor expectativa de seguir estudios superiores ${ }^{7}$.

La exposición inmediata y crónica a marihuana afecta también la capacidad de conducción con el riesgo de accidentes ${ }^{8}$. Un meta-análisis de encontró una asociación entre la intoxicación de cannabis y mayor riesgo de accidente de tránsito con heridos graves o muerte. Los conductores que consumen marihuana tienen dos a siete veces más probabilidades de ser responsables de accidentes en comparación con conductores que no usan drogas. Existe una relación entre concentración de THC en la sangre y rendimiento en conducción en estudios de simulación controlada.

En cuanto al riesgo de cáncer, en especial de pulmón no es claro. Fumar marihuana se asocia con inflamación de las vías respiratorias, aumento de la resistencia de vía aérea e hiperinsuflación pulmonar, asociaciones que son consistentes con el hecho que los fumadores regulares de marihuana son más propensos a bronquitis crónica. La competencia inmunológica del sistema respiratorio también pueden verse afectada por aumento de tasas de infecciones respiratorias y neumonía.

La marihuana además se ha asociado aumento de los riesgos de infarto de miocardio, accidente cerebrovascular, y ataques isquémicos transitorios durante la intoxicación aguda. Los efectos directos de los cannabinoides sobre diversos receptores diana, Receptores CB1 en los vasos sanguíneos arteriales y efectos indirectos sobre componentes vasoactivos expli- 
can los efectos perjudiciales de la marihuana sobre la resistencia vascular y la microcirculación coronaria

Es difícil evaluar el efecto de la exposición intrauterina a la marihuana, las madres suelen ser policonsumidoras en la experiencia nacional. El uso de marihuana durante la gestación en modelos animales sugiere que se alteran procesos normales de desarrollo cerebral.

La relación entre el consumo de cannabis por jóvenes y daño psicosocial es probable que sea multidimensional; un alto consumo de marihuana se relaciona con ingresos más bajos, mayor necesidad de asistencia socioeconómica, desempleo, comportamiento delictual y menor satisfacción con la vida.

En Chile todos los fármacos que ingresan al registro nacional de medicamentos deben cumplir con procedimientos estandarizados de aprobación exigidos por el Instituto de Salud Pública (ISP). Estos procedimientos, están destinados a proteger la salud de la población de efectos adversos y de prescripciones sin justificación. La preocupación actual es constatar cómo bajo la justificación del uso medicinal se desee legalizar la Marihuana a través de un proyecto de ley omitiendo las regulaciones sanitarias vigentes. Es necesario realizar investigaciones sobre los efectos de las políticas sobre la marihuana afectan la salud pública, allí las experiencias en países que han legalizado nos podrán ilustrar en el futuro próximo.

La OPS en su definición estrategias contra las drogas, propone a los países diferentes acciones para atacar el tráfico y el consumo, dependiendo de las realidades propias. Las políticas de drogas debieran incluir múltiples elementos y la discusión valórica debe tener un rol protagónico

El mayor problema reportado en Chile es el consumo, que incide directamente en el número de consumidores problemáticos, donde la mayoría son de sectores de alta vulnerabilidad social. La Declaración de los Derechos del Niño (ONU1959), fue un manifiesto con una determinada intención moral y ética para promover los derechos de los niños, no era un instrumento jurídicamente vinculante, como es el caso de la Convención de Derechos del Niño que en 1989 que se aprobó en la Asamblea Ge- neral de Naciones Unidas, ratificada por Chile en 1990 que asume el compromiso de asegurar a todos los niños y niñas derechos y principios, transformándose en uno de los tratados de derechos humanos más ratificados de todos los tiempos. En el Artículo 33 se expone que "Los Estados Partes adoptarán todas las medidas apropiadas, incluidas medidas legislativas, administrativas, sociales y educacionales, para proteger a los niños contra el uso ilícito de los estupefacientes y sustancias sicotrópicas enumeradas en los tratados internacionales pertinentes, y para impedir que se utilice a niños en la producción y el tráfico ilícitos de esas sustancias". Justamente debido a este componente del debate, es que debemos tener presente que más temprano que tarde entrarán en conflicto dos valores fundamentales: por un lado la libertad individual de consumir y por el otro es la protección de la salud.

\section{Referencias}

1.- Mönckeberg F: Los pro y contra de la legalizaciónde la marihuana. Rev Chil Pediatr 2014; 85 (2): 229-37.

2.- Babor T, Caulkins J, Edwards G, et al: La política de drogas y el bien público Washington DC. OPS, Oxford University Press (2010) ISBN: 978-92-75-33169-9.

3.- Volkow ND, Baler RB, Compton WM, Weiss SRB: Adverse Health Effects of Marijuana Use. N Engl J Med 2014; 370: 23, 5, 2219.

4.- Chen CY, Storr CL, Anthony JC: Early onset drug use and risk for drug dependence problems. Addict Behav 2009; 34: 319-22.

5.- Décimo Estudio Nacional de Drogas en Población Escolar Principales Resultados Nacionales Observatorio Chileno de Drogas Servicio Nacional para la Prevención y Rehabilitación del Consumo de Drogas y Alcohol (SENDA). Julio, 2014. http://www.senda.gob.cl/wpcontent/uploads/2014/07/decimo-escolares-2013.pdf

6.- Silins E, Horwood LJ, Patton GC for the Cannabis Cohorts Research Consortium: Young adult sequelae of adolescent cannabis use: an integrative analysis. Lancet Psychiatry 2014; 1: 286-93.

7.- Dörr A, Gorostegui ME, Viani S, Dörr BMP: Adolescentes consumidores de marihuana: implicaciones para la familia y la escuela. Salud Mental 2009; 32: 269-78.

8.- Brady JE, Li G: Trends in alcohol and other drugs detected in fatally injured drivers in the United States, 1999-2010. Am J Epidemiol 2014; 179: 692-9. 